\title{
DOA ESTIMATION USING A SPARSE UNIFORM LINEAR ARRAY WITH TWO CW SIGNALS OF CO-PRIME FREQUENCIES
}

\author{
Yimin D. Zhang ${ }^{\dagger}$, Moeness G. Amin ${ }^{\dagger}$, Fauzia Ahmad $^{\dagger}$, and Braham Himed ${ }^{\ddagger}$ \\ $\dagger$ Center for Advanced Communications, Villanova University, Villanova, PA 19085, USA \\ $\ddagger$ RF Technology Branch, Air Force Research Lab (AFRL/RYMD), WPAFB, OH 45433, USA
}

\begin{abstract}
In this paper, we propose the use of a sparse uniform linear array to estimate the direction-of-arrival (DOA) of more sources than the number of sensors by exploiting two continuous wave signals whose frequencies satisfy a certain coprime relationship. This extends the co-prime array and filter concept, which was developed in either the spectral or spatial domain, to a joint spatio-spectral domain, thereby achieving high flexibility in array structure design to meet the degreesof-freedom and system complexity constraints. The DOA estimation is implemented in the difference co-array context to avoid spatial undersampling, and group sparsity based compressive sensing techniques are used to determine the direction of signal arrivals.
\end{abstract}

Index Terms - Co-prime array, sparse array, DOA estimation, compressive sensing, group sparsity

\section{INTRODUCTION}

Co-array equivalence is an important concept in designing arrays with reduced redundancy and achieving high degreesof-freedom (DOFs) [1, 2]. The minimum redundancy array (MRA) is a well-known example of sparse linear array structures which, for a given number of elements, maximizes the number of consecutive virtual elements in the resulting in difference co-array [3]. Among others, the co-prime array was recently proposed as an attractive technique for sparse array construction [4]. A co-prime array utilizes a co-prime pair of uniform linear subarrays to achieve $O(M N)$ DOFs by using $M+N-1$ physical sensors. The increased DOFs can be exploited in direction-of-arrival (DOA) estimation and beamforming problems for improved performance [4]. In [5], the co-prime MUSIC technique was developed for DOA estimation of more uncorrelated sources than the number of physical sensors. This technique was extended in [6] and [7] by using sparsity-based techniques to consider the imperfect correlation and to achieve more efficient use of the available DOFs.

In this paper, we propose the use of a sparse uniform linear array (ULA) to construct a virtual co-prime array, which is

The work of Y. D. Zhang, M. G. Amin, and F. Ahmad was supported in part by a subcontract with Dynetics, Inc. for research sponsored by the Air Force Research Laboratory under Contract FA8650-08-D-1303, by a subcontract with Defense Engineering Corporation for research sponsored by the Air Force Research Laboratory under Contract FA8650-12-D-1376, and by the Office of Naval Research under Grant N00014-13-1-0061. then used to estimate the DOA information of a significantly higher number of sources than the number of sensors. This is achieved by exploiting two continuous-wave (CW) sinusoids whose frequencies satisfy a certain co-prime relationship. As such, the ULA acts as two virtual subarrays at these two frequencies, resulting in a similar structure to co-prime arrays. This extends the co-prime array and filter concept, which was developed in either the spectral or spatial domain, to a joint spatio-spectral domain, thereby achieving high flexibility in array structure design to meet the DOF and system complexity constraints. Unlike co-prime arrays wherein the numbers of elements and the interelement spacings have to satisfy the co-prime relationship, only the two frequencies are required to be co-prime in the proposed work. As a result, the proposed technique provides greater flexibility in system design and the capability of on-the-fly configuration adaptivity to the application environments. We also emphasize that, while the discussion in this paper is limited to two frequencies, the proposed technique can be easily extended to multiple frequencies to achieve even higher DOFs. Array processing exploiting dual- or multi-frequency $\mathrm{CW}$ signals has been considered for target localization by jointly estimating the target range and DOA information (e.g., [8, 9]).

From DOA estimation perspective, the challenges of the proposed structure lie in the fact that the reflection characteristics at the two frequencies differ, due to the differences in the propagation phase and possibly the target reflectivity. To account for such differences, the problem is formulated as a group (block) sparse problem where the targets, with different reflection characteristics, are located at the same spatial locations regardless of the operational frequencies. The group LASSO algorithm [10] is then employed to utilize the group sparsity for effective DOA estimation. Some wideband DOA estimation techniques have used the group sparsity concept (e.g., [11]). However, the primary objective therein was the exploitation of diversity in different frequencies in order to achieve improved robustness rather than higher DOFs.

Notations: We use lower-case (upper-case) bold characters to denote vectors (matrices). In particular, $\mathbf{I}_{N}$ denotes the $N \times N$ identity matrix. (.)* denotes complex conjugate, and $(.)^{T}$ and $(.)^{H}$ respectively denote the transpose and conjugate transpose of a matrix or vector, respectively. vec $(\cdot)$ denotes the vectorization operator that turns a matrix into a vector by concatenating all the columns, and $\operatorname{diag}(\mathbf{x})$ denotes a diagonal matrix with the elements of $\mathbf{x}$ constituting the diag- 
onal entries. $\|\cdot\|_{2}$ denotes the Euclidean $\left(l_{2}\right)$ norm of a vector, whereas $\|\cdot\|_{1}$ denotes the $l_{1}$ norm. $\mathrm{E}(\cdot)$ denotes the statistical expectation operator. $\bigotimes$ denotes the Kronecker product, and $\lfloor\cdot\rfloor$ denotes the largest integer not exceeding the argument.

\section{SYSTEM MODEL}

We consider a DOA estimation problem by simultaneously emitting two CW signals of frequencies $f_{1}$ and $f_{2}$ from a single transmit antenna or a phased array. The signals returned from $K$ unknown far-field targets are received at an $L$-element ULA, which has an interelement spacing of $d$.

For a CW waveform with frequency $f_{i}$, where $i$ is either 1 or 2 , the return signal from the $K$ far-field targets, located at DOAs $\theta_{k}, k=1,2, \ldots, K$, is expressed as

$$
\tilde{\mathbf{x}}_{i}(t)=\exp \left(j 2 \pi f_{i} t\right) \sum_{k=1}^{K} \rho_{i}^{(k)}(t) \mathbf{a}_{i}\left(\theta_{k}\right)+\tilde{\mathbf{n}}_{i}(t)
$$

where $\rho_{i}^{(k)}(t)$ is the reflection coefficient which does not vary with the receive antennas but is in general frequencydependent because the phase delay varies with the frequency, and the target reflectivity may also differ at the two frequencies. The target reflection coefficients are assumed to be time-varying due to, e.g., target motion or radar cross section (RCS) fluctuations, and are assumed to be uncorrelated for different targets. In addition, $\mathbf{a}_{i}\left(\theta_{k}\right)$ is the steering vector corresponding to $\theta_{k}$, expressed as

$$
\mathbf{a}_{i}\left(\theta_{k}\right)=\left[1, e^{-j \frac{2 \pi d}{\lambda_{i}} \sin \left(\theta_{k}\right)}, \ldots, e^{-j \frac{2 \pi(L-1) d}{\lambda_{i}} \sin \left(\theta_{k}\right)}\right]^{T},
$$

where $\lambda_{i}=c / f_{i}$ denotes the wavelength corresponding to $f_{i}$, and $c$ is the velocity of wave propagation. Furthermore, $\tilde{\mathbf{n}}_{i}(t)$ is the additive noise vector, whose elements are assumed to be spatially and temporally white.

After converting the received signal vector to baseband using the respective transmitted $\mathrm{CW}$ waveforms, followed by low-pass filtering, we obtain

$$
\mathbf{x}_{i}(t)=\sum_{k=1}^{K} \rho_{i}^{(k)}(t) \mathbf{a}_{i}\left(\theta_{k}\right)+\mathbf{n}_{i}(t)=\mathbf{A}_{i} \mathbf{d}_{i}(t)+\mathbf{n}_{i}(t),
$$

where $\mathbf{A}_{i}=\left[\mathbf{a}_{i}\left(\theta_{1}\right), \ldots, \mathbf{a}_{i}\left(\theta_{K}\right)\right]$ and $\mathbf{d}_{i}(t)=\left[\rho_{i}^{(1)}(t), \ldots\right.$, $\left.\rho_{i}^{(K)}(t)\right]^{T}$. We denote the noise variance at the filter output as $\sigma_{n}^{(i)}$.

In [12], a sufficient condition for alias-free DOA estimation is achieved by choosing the two frequencies to be coprime, i.e., their ratio equals the ratio between two co-prime numbers. In this paper, we consider the problem in the context of co-prime arrays as described in [4]. As such, we assume that $d$ is an integer multiple of the half-wavelength at both frequencies, i.e., $M_{i}=2 d / \lambda_{i}$ are integers for $i=1,2$. As such, the ULA is sparse (spatially undersampled) at each frequency. $M_{1}$ and $M_{2}$ are chosen to be co-prime, i.e., their greatest common divisor (GCD) is one. Without loss of generality, we assume $M_{1}<M_{2}$. In this case, we can rewrite the steering vectors in a frequency-independent form for better clarity,

$$
\mathbf{a}_{i}\left(\theta_{k}\right)=\left[1, e^{-j M_{i} \pi \sin \left(\theta_{k}\right)}, \ldots, e^{-j(L-1) M_{i} \pi \sin \left(\theta_{k}\right)}\right]^{T}
$$

As such, the DOA estimation problem is similar to the coprime arrays considered in $[5,7]$. Instead of a co-prime array consisting of two physical subarrays, we use a ULA with two sensing frequencies that satisfy a co-prime relationship. In addition, unlike in $[4,5,7]$ where the two uniform linear subarrays have different numbers of array sensors, the two uniform linear subarrays in the underlying problem have the same $L$ sensors. The co-array positions are listed as

$S=\left\{M_{1} l_{1} d_{0}, 0 \leq l_{1} \leq L-1\right\} \cup\left\{M_{2} l_{2} d_{0}, 0 \leq l_{2} \leq L-1\right\}$,

where $d_{0}$ denotes half-wavelength in a normalized frequency sense (i.e., no specific frequency is referred to). Note that the two subarrays align in the zeroth position and whenever $l / M_{2}$ is an integer. Therefore, the total number of unique array sensor positions is $2 L-1-\left\lfloor(L-1) / M_{2}\right\rfloor$.

\section{GROUP SPARSITY BASED DOA ESTIMATION}

While the DOA estimation problem considered here appears to be similar to that considered in $[5,7]$, the method developed therein cannot be readily applied to the underlying problem. A major distinction is that the target reflection coefficient, $\rho_{i}^{(k)}(t)$, generally differs at the two frequencies due to differences in their propagation phase delay and target reflectivity. As such, the phase term of the cross-correlation between $\mathbf{x}_{1}(t)$ and $\mathbf{x}_{2}(t)$ depends not only on the spatial angles, but also on the unknown phase difference in the reflection coefficients. This unknown phase difference prohibits the construction of a difference co-array from the estimated covariance matrix.

In this section, we first address the effect of the unknown phase difference in Section 3.1. In Section 3.2, we formulate the DOA estimation problem as a group sparsity based signal recovery problem, which is then solved using the group LASSO algorithm [10].

\subsection{Effect of Different Reflection Coefficients}

Denote $\mathbf{x}(t)=\left[\mathbf{x}_{1}^{T}(t), \mathbf{x}_{2}^{T}(t)\right]^{T}$. The covariance matrix of data vector $\mathbf{x}(t)$ is expressed as

$$
\mathbf{R}_{\mathbf{x x}}=\mathrm{E}\left[\mathbf{x}(t) \mathbf{x}^{H}(t)\right]=\left[\begin{array}{cc}
\mathbf{R}_{\mathbf{x}}^{(1)} & \mathbf{R}_{\mathbf{x x}}^{(1,2)} \\
\mathbf{R}_{\mathbf{x x}}^{(2,1)} & \mathbf{R}_{\mathbf{x x}}^{(2)}
\end{array}\right]
$$

which, in practice, is obtained by the sample average. In (6),

$$
\begin{aligned}
\mathbf{R}_{\mathbf{x x}}^{(i)} & =\mathrm{E}\left[\mathbf{x}_{i}(t) \mathbf{x}_{i}^{H}(t)\right]=\mathbf{A}_{i} \mathbf{R}_{\mathbf{s s}}^{(i)} \mathbf{A}_{i}^{H}+\sigma_{n}^{(i)} \mathbf{I}_{L} \\
& =\sum_{k=1}^{K} \sigma_{k}^{(i)} \mathbf{a}_{i}\left(\theta_{k}\right) \mathbf{a}_{i}^{H}\left(\theta_{k}\right)+\sigma_{n}^{(i)} \mathbf{I}_{L}
\end{aligned}
$$


and

$$
\begin{aligned}
\mathbf{R}_{\mathbf{x x}}^{(i, l)} & =\mathrm{E}\left[\mathbf{x}_{i}(t) \mathbf{x}_{l}^{H}(t)\right]=\mathbf{A}_{i} \mathbf{R}_{\mathbf{s s}}^{(i, l)} \mathbf{A}_{l}^{H} \\
& =\sum_{k=1}^{K} \sigma_{k}^{(i, l)} \mathbf{a}_{i}\left(\theta_{k}\right) \mathbf{a}_{l}^{H}\left(\theta_{k}\right),
\end{aligned}
$$

for $1 \leq i \neq l \leq 2$. In the above expressions, $\mathbf{R}_{\mathbf{s s}}^{(i)}=$ $\mathrm{E}\left[\mathbf{d}_{i}(t) \mathbf{d}_{i}^{H}(t)\right]=\operatorname{diag}\left(\left[\sigma_{1}^{(i)}, \ldots, \sigma_{K}^{(i)}\right]\right)$ is the source covariance matrix where $\sigma_{k}^{(i)}$ is real and positive, whereas $\mathbf{R}_{\mathbf{s s}}^{(i, l)}=$ $\mathrm{E}\left[\mathbf{d}_{i}(t) \mathbf{d}_{l}^{H}(t)\right]=\operatorname{diag}\left(\left[\sigma_{1}^{(i, l)}, \ldots, \sigma_{K}^{(i, l)}\right]\right)$. Note that $\sigma_{k}^{(i, l)}$ is in general a complex number. As such, the elements of $\mathbf{R}_{\mathbf{x x}}^{(i, l)}$, $i \neq l$, do not provide the phase progression information which is associated with the signal DOAs and the array configuration. Since phase progression information is a key requirement in constructing the difference co-array as in the co-prime arrays considered in $[4,5,7]$, existing techniques cannot be directly applied for the estimation of signal DOAs in the underlying problem.

\subsection{Proposed Technique}

Vectorizing $\mathbf{R}_{\mathbf{x x}}^{(i)}$ in (7) yields an $L^{2} \times 1$ vector

$$
\mathbf{z}^{(i)}=\operatorname{vec}\left(\mathbf{R}_{\mathbf{x x}}^{(i)}\right)=\tilde{\mathbf{A}}^{(i)} \mathbf{b}^{(i)}+\sigma_{n}^{(i)} \mathbf{i},
$$

where $\tilde{\mathbf{A}}^{(i)}=\left[\tilde{\mathbf{a}}_{i}\left(\theta_{1}\right), \ldots, \tilde{\mathbf{a}}_{i}\left(\theta_{K}\right)\right], \tilde{\mathbf{a}}_{i}\left(\theta_{k}\right)=\mathbf{a}_{i}^{*}\left(\theta_{k}\right) \otimes \mathbf{a}_{i}\left(\theta_{k}\right)$, $\mathbf{b}^{(i)}=\left[\sigma_{1}^{(i)}, \ldots, \sigma_{K}^{(i)}\right]^{T}$, and $\mathbf{i}=\operatorname{vec}\left(\mathbf{I}_{L}\right)$. Note that $\tilde{\mathbf{a}}_{i}\left(\theta_{k}\right)$ provides phase information of $\theta_{k}$ for array sensor lags that are integer multiples of $M_{i} d_{0}$, where $i=1,2$.

Similarly, vectorizing $\mathbf{R}_{\mathbf{x x}}^{(i, l)}$ in (8), we obtain

$$
\mathbf{z}^{(i, l)}=\operatorname{vec}\left(\mathbf{R}_{\mathbf{x x}}^{(i, l)}\right)=\tilde{\mathbf{A}}^{(i, l)} \mathbf{b}^{(i, l)},
$$

where $\tilde{\mathbf{A}}^{(i, l)}=\left[\tilde{\mathbf{a}}_{i, l}\left(\theta_{1}\right), \ldots, \tilde{\mathbf{a}}_{i, l}\left(\theta_{K}\right)\right], \tilde{\mathbf{a}}_{i, l}\left(\theta_{k}\right)=\mathbf{a}_{i}^{*}\left(\theta_{k}\right) \otimes$ $\mathbf{a}_{l}\left(\theta_{k}\right), \mathbf{b}^{(i, l)}=\left[\sigma_{1}^{(i, l)}, \ldots, \sigma_{K}^{(i, l)}\right]^{T} . \tilde{\mathbf{a}}_{i, l}\left(\theta_{k}\right)$ provides phase information of $\theta_{k}$ for array sensor lags that are not necessarily integer multiples of $M_{i} d_{0}$ for $i=1,2$. As we described earlier, the elements of $\mathbf{b}^{(i, l)}$ generally take complex values. Also note that only $\mathbf{R}_{\mathbf{x} x}^{(1,2)}$ is considered in the sequel because $\mathbf{R}_{\mathbf{x x}}^{(2,1)}=\left[\mathbf{R}_{\mathbf{x x}}^{(1,2)}\right]^{*}$.

In (9) and (10), the elements of $\mathbf{b}^{(i)}$ and $\mathbf{b}^{(i, l)}$ take different values but the two vectors have a common support corresponding to the $K$ targets that are sparsely located in space. The DOA estimation problem, therefore, can be considered as a group sparse problem of locating the non-zero entries of $\mathbf{b}^{(i)}$ and $\mathbf{b}^{(i, l)}$ in the space. A number of methods, such as the group LASSO [10] and the block orthogonal matching pursuit (BOMP) [13], have been developed to solve this type of problem. In this paper, the group LASSO is used.

Stacking vectors $\mathbf{z}^{(1)}, \mathbf{z}^{(2)}$, and $\mathbf{z}^{(1,2)}$ as $\mathbf{z}=\left[\left(\mathbf{z}^{(1)}\right)^{T}\right.$, $\left.\left(\mathbf{z}^{(2)}\right)^{T},\left(\mathbf{z}^{(1,2)}\right)^{T}\right]^{T}$, we combine (9) and (10) into a single expression as,

$$
\mathbf{z}=\tilde{\mathbf{A}} \mathbf{b}+\tilde{\mathbf{I}} \sigma_{n},
$$

where $\mathbf{b}=\left[\left(\mathbf{b}^{(1)}\right)^{T},\left(\mathbf{b}^{(2)}\right)^{T},\left(\mathbf{b}^{(1,2)}\right)^{T}\right]^{T}, \tilde{\mathbf{A}}$ is a block diagonal matrix consisting of $\tilde{\mathbf{A}}^{(1)}, \tilde{\mathbf{A}}^{(2)}$, and $\tilde{\mathbf{A}}^{(1,2)}$. In addition, $\sigma_{n}=\left[\sigma_{n}^{(1)}, \sigma_{n}^{(2)}\right]^{T}$, and $\tilde{\mathbf{I}}$ is a $3 L^{2} \times 2$ matrix whose first column is $\left[\mathbf{i}^{T}, \mathbf{0}^{T}, \mathbf{0}^{T}\right]^{T}$, where $\mathbf{0}$ denotes the all zero vector of dimension $L^{2} \times 1$, and the second column is $\left[\mathbf{0}^{T}, \mathbf{i}^{T}, \mathbf{0}^{T}\right]^{T}$.

The expression in (11) can be replaced by the sparse signal representation in the following equivalent format,

$$
\mathbf{z}=\tilde{\mathbf{B}} \mathbf{r}+\tilde{\mathbf{I}} \sigma_{n}=\tilde{\mathbf{B}}^{o} \mathbf{r}^{o},
$$

where $\mathbf{r}$ is a $3 Q \times 1$ vector with $Q$ denoting the size of search grid in spatial angles, and $\tilde{\mathbf{B}}$ is a counterpart of $\tilde{\mathbf{A}}$ with an expanded dimension corresponding to the entire search grid. Note that $\mathbf{r}$ has only $3 K$ non-zero entries which correspond to the elements of $\sigma_{k}^{(1)}, \sigma_{k}^{(2)}$, and $\sigma_{k}^{(1,2)}$ at their respective positions, representing the target DOAs. The elements of $\mathbf{r}$ satisfy a group sparse model, i.e., the non-zero entries corresponding to the different frequency combinations share the same support. In addition, $\mathbf{B}^{o}=[\tilde{\mathbf{B}}, \tilde{\mathbf{I}}]$ and $\mathbf{r}^{o}=\left[\mathbf{r}^{T}, \sigma_{n}^{T}\right]^{T}$ were used in the above expression for notational simplicity.

The estimation of $\mathbf{r}$ is obtained as the first $3 Q$ elements of $\mathbf{r}^{o}$ by solving the following minimization problem,

$$
\hat{\mathbf{r}}^{o}=\arg \min _{\mathbf{r}^{o}}\left\|\xi\left(\mathbf{r}^{o}\right)\right\|_{1} \quad \text { s.t. } \quad\left\|\mathbf{z}-\mathbf{B}^{o} \mathbf{r}^{o}\right\|_{2}<\epsilon,
$$

where $\epsilon$ is a user-specific bound, and $\xi($.$) is an operation that$ obtains the $l_{2}$ norm of the three-element entries corresponding to each spatial position. As such, $\xi\left(\mathbf{r}^{o}\right)$ returns a $Q \times 1$ vector. Equivalently, the above expression can be reformulated as

$$
\hat{\mathbf{r}}^{o}=\arg \min _{\mathbf{r}^{o}}\left[\frac{1}{2}\left\|\mathbf{z}-\mathbf{B}^{o} \mathbf{r}^{o}\right\|_{2}+\lambda_{t}\left\|\xi\left(\mathbf{r}^{o}\right)\right\|_{1}\right],
$$

where $\lambda_{t}$ is a regularization parameter. The group LASSO algorithm finds the group sparse solution by solving the minimization problem in (14).

\section{SIMULATION RESULTS}

We use a set of parameters that resemble those in [7]. Two co-prime subarrays with 5 and 6 sensors, respectively, were used in [7], resulting in a total number of 10 physical array sensors in the co-prime array. In this paper, we consider a ULA of $L=6$ antenna sensors, and $M_{1}=5$ and $M_{2}=6$ are assumed. $K=17$ targets, which are uniformly distributed between $-50^{\circ}$ and $50^{\circ}$, are assumed. The noise power at the two frequencies are assumed to be the same, and the input SNRs for all sources are assumed to be identical. The number of snapshots is 500, and the grid size for space angle search is $0.1^{\circ}$. The phase difference between the received signal corresponding to the two frequencies is independently and uniformly distributed over $[0,2 \pi]$.

We first show the estimated spatial spectrum using the technique described in [7] based on conventional LASSO. The input SNR is $0 \mathrm{~dB}$. It is clear that the 17 targets are resolved only when there is no phase difference between the signals observed at the two frequencies. The estimated spatial spectrum becomes very poor when such phase difference does exist.

The estimated spectrum from the group LASSO algorithm is shown in Fig. 2, where the input SNR levels of $-10 \mathrm{~dB}$ and 
$0 \mathrm{~dB}$ are considered. It is seen that all 17 sources are correctly or closely identified. However, spurious peaks are observed, particularly at low SNR, due to the sidelobe effect as the result of the sparsity. This problem is inherent to the co-prime array and was also observed in [7]. Some approaches for sidelobe reduction in co-prime arrays have been reported (e.g., [14]).

While the parameters are similar to those considered in [7], the array considered here has slightly higher DOFs because 6 physical sensors are shared by both co-prime subarrays (whereas in [7] the two subarrays respectively have 5 and 6 elements). In addition, we used all of the available difference lags for DOA estimation, whereas only the consecutive lags were used in [7].

In the proposed technique, co-prime arrays are not explicitly reconstructed as in $[5,7]$. Nevertheless, it is important to note the importance of using co-prime frequencies to maximize the DOFs. To demonstrate this fact, we show in Fig. 3 the results when we choose $M_{1}$ and $M_{2}$ not to be co-prime. Specifically, $M_{1}=4$ and $M_{2}=6$ are used in this example, where the input SNR is $0 \mathrm{~dB}$, and the other parameters are kept unchanged. It is clear from Fig. 3 that this configuration yields poor estimates of the spatial spectrum.

\section{CONCLUSION}

We have proposed the use of a sparse uniform linear array for effective DOA estimation by exploiting two continuous-wave sinusoids with co-prime frequencies. The difference co-array concept is used to avoid the spatial undersampling effect. The group LASSO algorithm is used to estimate the spatial signal spectrum under common support of the target reflection coefficients at different frequencies. The superior performance of the proposed technique is verified using simulation results.

\section{REFERENCES}

[1] R. T. Hoctor and S. A. Kassam, "The unifying role of the co-array in aperture synthesis for coherent and incoherent imaging," Proc. IEEE, vol. 78, no. 4, pp. 735-752, April 1990.

[2] F. Ahmad and S. A. Kassam, "Performance analysis and array design for wide-band beamformers," J. Electronic Imaging, vol. 7, no. 4, pp. 825-838, Oct. 1998.

[3] A. Moffet, "Minimum-redundancy linear arrays," IEEE Trans. Antennas Propagat., vol. 16, no. 2, pp. 172-175, March 1968.

[4] P. P. Vaidyanathan and P. Pal, "Sparse sensing with co-prime samplers and arrays," IEEE Trans. Signal Proc., vol. 59, no. 2, pp. 573-586, Feb. 2011.

[5] P. Pal and P. P. Vaidyanathan, "Coprime sampling and the MUSIC algorithm," in Proc. IEEE Digital Signal Proc. Workshop and IEEE Signal Proc. Education Workshop, Sedona, AZ, Jan. 2011.

[6] P. Pal and P. P. Vaidyanathan, "On application of LASSO for sparse support recovery with imperfect correlation awareness," in Proc. Asilomar Conf. Signals, Systems and Computers, Pacific Grove, CA, Nov. 2012.

[7] Y. D. Zhang, M. G. Amin, and B. Himed, "Sparsity-based DOA estimation using co-prime arrays," in Proc. IEEE ICASSP, Vancouver, Canada, May 2013.

[8] Y. Zhang, M. G. Amin, and F. Ahmad, "A novel approach for multiple moving target localization using dual-frequency radars and timefrequency distributions," in Proc. Annual Asilomar Conf. Signals, Systems, and Compters, Pacific Grove, CA, Nov. 2007.

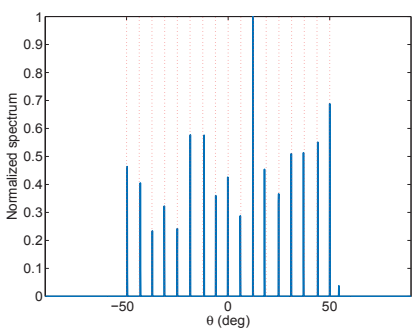

(a) no phase difference

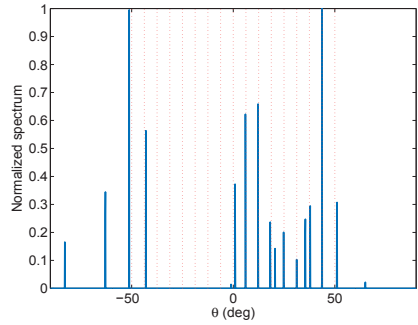

(b) with phase difference
Fig. 1. Spatial spectrum obtained from LASSO-based technique for cases without and with random phase difference between the two frequencies (input $\mathrm{SNR}=0 \mathrm{~dB}$ ).

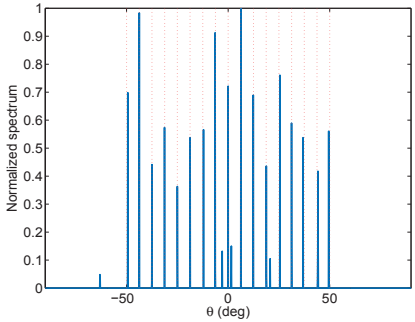

(a) input $\mathrm{SNR}=-10 \mathrm{~dB}$

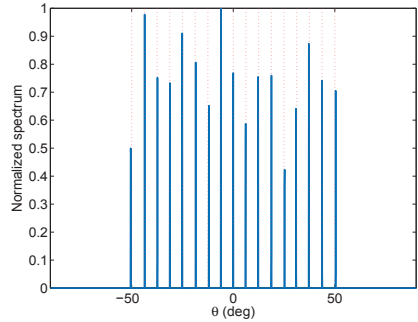

(b) input $\mathrm{SNR}=0 \mathrm{~dB}$
Fig. 2. Spatial spectrum estimated using group LASSO.

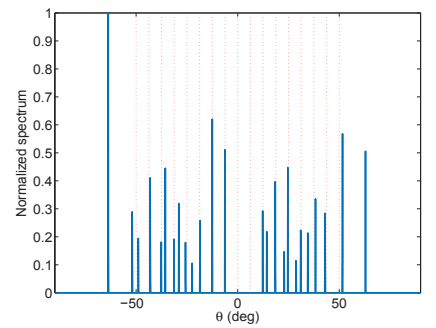

Fig. 3. Spatial spectrum with $M_{1}=4$ and $M_{2}=6$ (input $\mathrm{SNR}=0 \mathrm{~dB})$.

[9] Y. D. Zhang, X. Li, and M. G. Amin, "Target localization in multipath environment through the exploitation of multi-frequency array," in Proc. Int. Waveform Div. Design Conf., Niagara Falls, Canada, Aug. 2010.

[10] M. Yuan and Y. Lin, "Model selection and estimation in regression with grouped variables', J. Royal Stat. Soc., Series B, vol. 68, no. 1, pp. 4967, 2007.

[11] J-A. Luo, X-P. Zhang, and Z. Wang, "A new subband information fusion method for wideband DOA estimation using sparse signal repesentation," in Proc. IEEE ICASSP, Vancouver, Canada, May 2013.

[12] M. G. Amin, "Sufficient conditions for alias-free direction of arrival estimation in periodic spatial spectra," IEEE Trans. Antennas Propagat., vol. 41, no. 4, pp. 508-511, April 1993.

[13] Y. Eldar, P. Kuppinger, and H. Bolcskei, "Block-sparsity signals: Uncertainty relations and efficient recovery," IEEE Trans. Signal Proc., vol. 58, no. 6, pp. 3042-3054, June 2010.

[14] K. Adhikari, J. Buck, and K. Wage, "Beamforming with extended coprime sensor arrays," in Proc. IEEE ICASSP, Vancouver, Canada, May 2013. 